\title{
The impact of nocturnal disturbances on daily quality of life in patients with Parkinson's disease
}

\author{
This article was published in the following Dove Press journal: \\ Neuropsychiatric Disease and Treatment \\ 6 August 2015 \\ Number of times this article has been viewed
}

\section{Rwei-Ling $\mathrm{Yu}^{\prime}$ \\ Chun-Hsiang Tan² \\ Ruey-Meei $\mathrm{Wu}^{3}$}

'Institute of Behavioral Medicine, College of Medicine, National Cheng Kung University, Tainan, Taiwan; ${ }^{2}$ Wolfson Centre for Age Related Diseases, King's College London, London, UK; ${ }^{3}$ Department of Neurology, National Taiwan University Hospital, College of Medicine, National Taiwan University, Taipei, Taiwan
Correspondence: Ruey-Meei Wu Department of Neurology, National Taiwan University Hospital, No 7, Chung Shan South Road, Taipei 10002, Taiwan

Tel +886223123456 ext 65337

Fax +8862234I 8395

Email robinwu@ntu.edu.tw
Objectives: The aims of this study were to explore nocturnal disturbances in patients with Parkinson's disease (PD) and to assess their impact on quality of life (QoL).

Methods: A total of 211 patients with PD were recruited for this study, and each participant was evaluated using the mini-mental state examination, PD sleep scale - second version (PDSS-2), pittsburgh sleep quality index (PSQI), PD QoL questionnaire (PDQ), Epworth sleepiness scale, Hoehn and Yahr (H\&Y) staging, and unified Parkinson's disease rating scale (UPDRS). Multiple regression analyses were performed to determine the contribution of the predictive variables on QoL.

Results: There were $56.4 \%$ males (mean age: 64.08 years; disease duration: 6.02 years; H\&Y stage: 2.25 ; and UPDRS: 33.01 ) in this study. Our patients' actual sleep time was 5.96 \pm 1.16 hours and the average sleep efficiency was $82.93 \% \pm 12.79 \%$. Up to $64.4 \%$ of patients were classified as "poor" sleepers and $23.8 \%$ suffered from daytime sleepiness. The final stepwise regression model revealed that UPDRS parts I and II, the sleep disturbance and daytime dysfunction components of the PSQI, the PD symptoms at night subscale of the PDSS-2, and the levodopa equivalent dose were significant predictors of the PDQ score $\left(R^{2}=53, F_{7,165}=28.746 ; P<0.001\right)$.

Conclusion: Most of the PD patients have sleep problems, and nearly one-quarter of them have abnormal daytime somnolence. The nocturnal disturbances were found to result in worse QoL in PD patients. Ethnicity-specific effects of susceptibility to sleep disturbances were discussed, and these results also highlighted the direction for further studies to explore when examining effective management programs toward these disturbances.

Keywords: sleep, non-motor symptoms, Parkinson's disease, quality of life

\section{Introduction}

Parkinson's disease (PD) is classically characterized by its motor deficits, including resting tremor, rigidity, bradykinesia, and postural instability. While motor symptoms are the main clinical features of PD, increasing evidence shows that PD patients also have non-motor symptom disturbances, ${ }^{1}$ such as sleep disturbances, ${ }^{2}$ cognitive dysfunction, ${ }^{3}$ and impairments in social cognition. ${ }^{4,5}$

Sleep disturbances and nocturnal disturbances are common in patients with $\mathrm{PD} ; 6,7$ community-based studies have reported that about $60 \%$ of PD patients had a sleep disorder. ${ }^{2}$ Patients with PD often have frequent early awakening, sleep fragmentation, insomnia, nocturnal cramps, and nightmares, as well as impaired motor function during the night. Excessive daytime sleepiness (EDS) is defined as inappropriate and undesirable sleepiness during the waking hours. Evidence shows that EDS is 15-fold more frequent in PD patients than in age-matched healthy controls, and it affects up to $50 \%$ of PD patients. ${ }^{8}$ Moreover, EDS results in distractions and impairs driving or working performance. Although sleep disturbance is one of the important non-motor symptoms 
in PD, thus far, few studies have been conducted to evaluate the prevalence of daytime and night-time sleep disturbances in Oriental populations. In Asia, a cross-sectional study from Japan showed that $38 \%$ of patients have poor sleep quality ${ }^{8}$ and about $20 \%$ of Asian patients have daytime sleepiness (Japan: 21.3\% and Singapore: 19.9\%).,

Sleep and its associated factors in PD patients have been examined in several studies; ${ }^{10-14}$ however, the predictive factors vary and are inconsistent, and most studies have focused on western populations. Sleep disorders have been shown to significantly impair individuals' quality of life (QoL); ${ }^{7,11,15}$ nevertheless, few of these works have examined the detailed aspects of sleep disturbances, and they have not investigated the impact of both daytime and night-time sleep disturbances on QoL in relation to motor symptoms.

The aims of this study were to investigate the prevalence of daytime somnolence and night-time sleep disturbances, as well as to explore the predictive factors for sleep quality. Furthermore, we also aimed to explore the impact of sleep disturbances on QoL.

\section{Methods}

\section{Participants}

A total of $211 \mathrm{PD}$ patients were included. The patients were recruited from the neurology outpatient clinics at the National Taiwan University Hospital, and a clinical diagnosis of PD was made according to the United Kingdom Parkinson's Disease Society Brain Bank criteria. ${ }^{16}$ The inclusion criteria for patients were as follows: patients should be mentally able to complete interviews, self-report questionnaires, and clinical examinations (as assessed by the mini-mental state examination [MMSE] with a score $\geq 24$ ). The researchers interviewed each participant and administered the questionnaires in a face-to-face manner. The patients filled the questionnaires by themselves and the investigator provided assistance if needed. The exclusion criteria were as follows: patients with atypical Parkinsonism, as well as those with a history of brain surgery, other neurologic disorders, and psychiatric disorders. All participants provided written informed consent prior to enrollment, which was in accordance with the ethical standards outlined in the 1964 Declaration of Helsinki. This study was approved by the Ethical Research Committee of National Taiwan University Hospital.

\section{Measures}

Each patient received evaluations with the MMSE to measure their general mental ability, as well as with the Hoehn and Yahr (H\&Y) Staging scale and the unified Parkinson's disease rating scale (UPDRS) ${ }^{17}$ to measure the severity and characteristics of their motor symptoms. The questionnaires used in this study were the Pittsburgh sleep quality index (PSQI) - Taiwan form, ${ }^{18}$ the 39-item PD QoL questionnaire (PDQ), ${ }^{19}$ the Chinese version of the Epworth sleepiness scale (ESS), ${ }^{20}$ and the PD sleep scale - second version (PDSS-2)..$^{21}$

The PSQI measures different aspects of sleep disturbances, including sleep latency, duration, and habitual sleep efficiency, subjective sleep quality, the use of sleep medication; and daytime dysfunction over the past month. Seven sub-scores were summed up to yield a total score ranging from 0 to 21, and poor sleep quality was defined as a PSQI score $>5 .^{22}$ The PSQI - Taiwan form was translated into traditional Chinese; the Chinese version is also a reliable (Cronbach's $\alpha=0.79$; test-retest reliability $=0.91$ ) and valid (good construct and convergent validity) instrument for measuring sleep quality among Taiwanese patients. ${ }^{18}$

The patients' daily QoL was assessed by the Chinesetranslated PDQ. ${ }^{19}$ The PDQ includes 39 items, and previous factor analyses support an eight-factor model: mobility, activities of daily living, emotional well-being, stigma, social support, cognition, communication, and bodily discomfort. Each item on the mental health subscale is scored on a 5-point categorical scale. This scale was translated into Chinese; it has been shown to have good internal consistency reliability (Cronbach's $\alpha=0.80-0.96$ ) and it has been validated specially for people with PD. ${ }^{19}$

The ESS is an effective instrument used to measure average daytime sleepiness. Patients provide ratings on how likely it is that he/she would have a catnap in eight different situations. The answers are scored on a scale with responses ranging from 0 to 3 , with 0 being "would never doze" and 3 being a "high chance of dozing". A sum of 10 or more from the eight individual scores generally indicates pathological sleepiness. The Chinese version of the ESS showed acceptable reliability (Cronbach's $\alpha=0.81$ ), and it appears to be valid and sensitive to clinical change. ${ }^{20}$

The PDSS-2 assesses the patients' sleep disturbances and it consists of 15 questions pertaining to the following domains: overall quality of a night's sleep, sleep onset and maintenance insomnia, nocturnal restlessness, nocturnal psychosis, nocturia, nocturnal motor symptoms, sleep refreshment, and daytime dozing. ${ }^{21}$ Each item was rated by the patients using one of five categories, with responses ranging from 0 (never) to 4 (very frequent). The PDSS-2 total score ranges from 0 (no disturbance) to 60 (maximum nocturnal disturbance); three factors (ie, motor symptoms 
at night, PD symptoms at night, and disturbed sleep) are represented in this scale.

\section{Statistical analysis}

Descriptive statistics for sleep problems, as well as clinical and demographic variables were calculated. Correlations between the study variables were made, and logistic regression analyses were applied to determine the predictors of sleep quality at night. The effect size (Cohen's $d$ ) was reported to show the magnitude of the difference between groups. The multiple linear regression analyses were performed in order to determine the relative strength of the effects of age, sex, disease duration, motor characteristics, and sleep disturbances on QoL; the variables were entered into the analyses in this order using the enter method. SPSS version 21.0 was used for the statistical analyses.

\section{Results}

A total of 211 PD patients were included (Table 1); their mean actual sleep time was $5.96 \pm 1.16$ hours, and their sleep efficiency was $82.93 \% \pm 12.79 \%$. Up to $64.4 \%$ of patients were classified as "poor" sleepers (PSQI $>5$ ) and $23.8 \%$ of patients were classified as having EDS (ESS $\geq 10$ ) (Figure 1).

Table I Demographic and clinical characteristics of all PD patients $(n=2 \mid I)$

\begin{tabular}{lll}
\hline & Mean & SD \\
\hline Sex, \% male & 56.4 & \\
Age, y & 64.08 & 9.44 \\
Age of onset, y & 57.97 & 10.20 \\
Education, y & 12.07 & 3.96 \\
Disease duration, y & 6.02 & 4.53 \\
Hoehn and Yahr stages & 2.25 & 0.84 \\
I & $17.8 \%$ & \\
2 & $47.6 \%$ & \\
3 & $26.4 \%$ & \\
4 & $8.2 \%$ & 1.78 \\
UPDRS part I (mentality) & 2.08 & 5.71 \\
UPDRS part II (daily activities) & 8.33 & $1 \mathrm{I} .57$ \\
UPDRS part III (motor) & 22.56 & 414.42 \\
LED (mg/day) & 696.94 & 1.58 \\
MMSE & 28.42 & $3.5 \mathrm{I}$ \\
PSQI global score & 7.23 & 5.04 \\
ESS & 5.85 & 6.58 \\
PDSS-2 total score & 12.02 & 2.55 \\
Factor I, motor symptoms at night & 2.50 & 2.78 \\
Factor 2, PD symptoms at night & 2.56 & 3.59 \\
Factor 3, disturbed sleep & 6.96 & 26.64 \\
PDQ & 37.64 & Parkinss \\
\hline
\end{tabular}

Abbreviations: PD, Parkinson's disease; y, years; UPDRS, unified Parkinson's disease rating scale; LED, levodopa equivalent dose; MMSE, mini-mental state examination; PSQI, Pittsburgh sleep quality index; ESS, Epworth sleepiness scale; PDSS-2, PD sleep scale - second version; PDQ, PD Quality of life questionnaire; $\mathrm{SD}$, standard deviation.
The percentage of each PDSS-2 item is shown in Figure 2. There were no significant differences in terms of sex, age, age of onset, stage, MMSE score, and levodopa equivalent dose (LED) between poor and good PD sleepers (Table 2). Sleep latency was significantly longer, and sleep time and sleep efficiency were worse in poor PD sleepers than in good sleepers. Two components of the PDSS-2 (motor symptoms at night and disturbed sleep) were significantly higher in poor than in good sleepers.

In the logistic regression model, subjective sleep quality, difficulties falling asleep, restlessness of the legs or arms at night, and the urge to move legs or arms at bedtime can predict whether the patient is a poor sleeper (Table 3 ). Significant and strong correlations were found between PDQ and the following variables: PDSS-2 ( $r=0.53)$, as well as UPDRS part I ( $r=0.50)$ and part II $(r=0.56)$. Moderate correlations were found between PDQ and disease duration ( $r=0.43)$, H\&Y stages $(r=0.43)$, LED $(r=0.37)$, and PSQI ( $r=0.40$ ) (Table 4$)$. In this multiple linear regression model, PDQ served as the dependent variable, and the variables showing significant bivariate correlations served as the independent variables. Seven independent variables accounted for 53\% of the variance in the PDQ with high statistical significance $\left(F_{7,165}=28.746 ; P<0.001\right)$ (Table 5). These variables included: UPDRS parts I and II, the sleep disturbance and daytime dysfunction components of the PSQI, the PD symptoms at night subscale of the PDSS-2 (eg, the pain score in the arms or legs, and uncomfortable and immobility at night), and the LED.

\section{Discussion}

Up to $56 \%$ of our patients were classified as "poor" sleepers during the nighttime, and about $24 \%$ had daytime sleepiness. The prevalence rates of daytime sleepiness in America and Europe were reported to be $40 \%{ }^{11,23}$ and $32 \%,{ }^{24,25}$ respectively. The EDS prevalence rate in Asian populations was reported to be $22 \%{ }^{8,9}$ when compared with western populations, the prevalence of EDS in Taiwan is relatively low. This discrepancy suggests that genetic or other factors, such as lifestyle differences across different cultures, may play an etiologic role. Given that the ESS has good psychometric properties when measuring PD patients' daytime somnolence, ${ }^{26}$ it has been used in many different countries. Using the ESS to measure daytime sleepiness might underestimate the prevalence of sleep disturbances in Taiwan because some items on the ESS are not suitable for our patients. For example, one item on the ESS measures one's sleep propensity while driving. The public transportation system is well established 
A

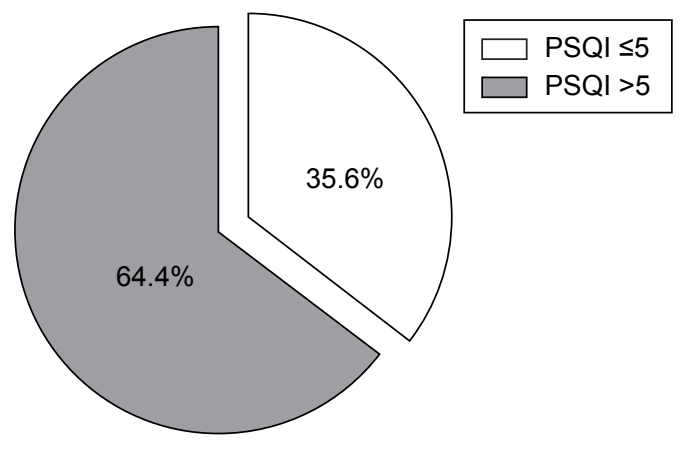

B

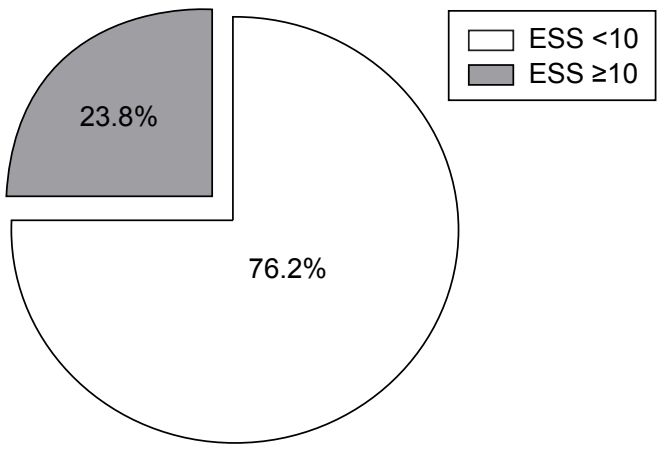

Figure I The proportion of $(\mathbf{A})$ poor sleeper (PSQI $>5$ ) and good sleeper and $(\mathbf{B})$ patients with (ESS $\geq 10$ ) and without excessive daytime sleepiness. Abbreviations: PSQI, Pittsburgh sleep quality index; ESS, Epworth sleepiness scale.

and user friendly for the elderly in our city; therefore, few of our patients drive by themselves. Around 30\% (range: $19.9 \%-40.6 \%)^{8,9,11,23-25}$ of PD patients worldwide suffer from daytime sleepiness. Since studies have previously focused on western populations, it is unclear whether there are any ethnicity-specific effects of susceptibility to somnolence. In the future, taking cultural differences into consideration, while developing a scale to measure somnolence in PD patients, should be important.

At night, more than half of our patients experience poor sleep, which is consistent with the findings from western studies (the Netherlands, 58.8\% $\%^{27}$ and France, 65.3\% ${ }^{25}$ ). The prevalence of poor sleepers in our study was higher than that of Japan. ${ }^{8}$ This discrepancy may be due to patients' recruitment. Cognitive functions were considered in our study, and our previous studies have shown that not all PD patients are accurately aware of their conditions. ${ }^{28} \mathrm{We}$ used the MMSE to exclude patients with dementia; as such, patients with dementia or those that were unaware of their conditions were not included. As compared with other studies, ${ }^{8,27}$ our patients have the shortest sleep duration, spending 7.2 hours in bed every night; however, they only experience 5.9 hours of actual sleep, on average. We suggest that teaching patients strategies to enhance their sleep efficiency is crucial for PD patients.

Western studies showed that the prevalence of nocturnal disturbances was around $60 \%-98 \%$ in PD patients. ${ }^{6,7}$ Our results found that more than $90 \%$ of Oriental PD patients were

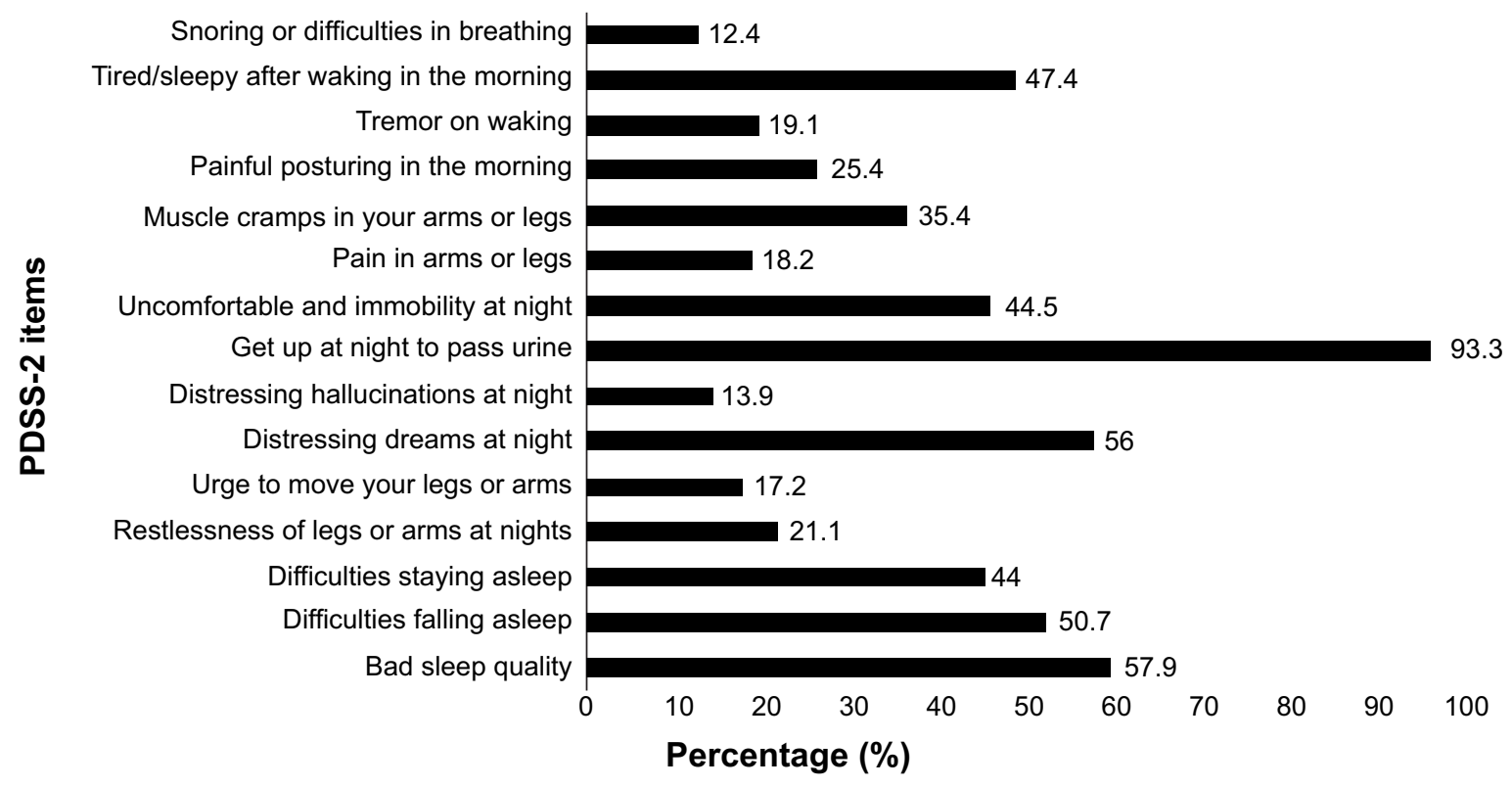

Figure 2 The histogram of the PDSS-2 percentage.

Abbreviation: PDSS-2, Parkinson's disease sleep scale - second version. 
Table 2 Characteristics of good and bad sleepers*

\begin{tabular}{|c|c|c|c|c|}
\hline & Good sleeper (PSQI $\leq 5)$ & Poor sleeper (PSQI $>5$ ) & $P$-value & Effect size $^{a}$ \\
\hline Sex, \% male & 61.1 & 54.6 & 0.23 & - \\
\hline Age, y & $63.20(9.64)$ & 64.5 I (9.24) & 0.34 & - \\
\hline Age of onset, $y$ & $57.13(10.28)$ & $58.30(10.18)$ & 0.43 & - \\
\hline Education, y & $12.67(3.94)$ & II.7| (3.90) & 0.07 & - \\
\hline Disease duration, $y$ & $5.93(4.66)$ & $6.15(4.56)$ & 0.63 & - \\
\hline Stages & $2.08(0.85)$ & $2.32(0.83)$ & 0.08 & - \\
\hline MMSE & $28.47(1.67)$ & $28.42(1.55)$ & 0.58 & - \\
\hline LED (mg/day) & $639.2(375.5)$ & $743.4(435.8)$ & 0.09 & - \\
\hline Fall asleep time & $10.37(7.65)$ & $27.91(36.20)$ & 0.00 & 0.67 \\
\hline Actual sleep time & $6.65(0.9631)$ & $5.59(1.11)$ & 0.00 & 1.02 \\
\hline Sleep efficiency & $92.03(6.76)$ & $78.24(12.97)$ & 0.00 & 1.33 \\
\hline UPDRS part I (mentality) & $1.51(1.19)$ & $2.42(1.97)$ & 0.00 & 0.56 \\
\hline UPDRS part II (daily activities) & $7.21(5.19)$ & $8.94(5.98)$ & 0.07 & - \\
\hline UPDRS part III (motor) & $21.38(11.65)$ & $22.86(11.38)$ & 0.39 & - \\
\hline PSQI total score & $3.65(1.20)$ & $9.22(2.7 I)$ & 0.00 & 2.66 \\
\hline PDSS-2 total score & $8.69(5.26)$ & $13.9 \mid(6.58)$ & 0.00 & 0.88 \\
\hline Factor I, motor symptoms at night & I.73 (2.03) & $2.92(2.73)$ & 0.00 & 0.49 \\
\hline Restlessness of legs or arms at nights & $0.10(0.30)$ & $0.50(0.99)$ & 0.00 & 0.55 \\
\hline Distressing dreams at night & $0.72(1.04)$ & $1.17(1.19)$ & 0.00 & 0.40 \\
\hline Painful posturing in the morning & $0.24(0.80)$ & $0.48(0.82)$ & 0.00 & 0.30 \\
\hline Factor 2, PD symptoms at night & $1.99(2.50)$ & $2.84(2.90)$ & 0.05 & - \\
\hline Distressing hallucinations at night & $0.11(0.55)$ & $0.36(0.87)$ & 0.01 & 0.34 \\
\hline Pain in arms or legs & $0.18(0.70)$ & $0.4 \mathrm{I}(0.95)$ & 0.02 & 0.28 \\
\hline Factor 3, disturbed sleep & $4.97(2.69)$ & $8.16(3.56)$ & 0.00 & 1.01 \\
\hline Bad sleep quality & $0.54(0.91)$ & $1.52(1.24)$ & 0.00 & 0.90 \\
\hline Difficulties falling asleep & $0.30(0.54)$ & $1.28(1.26)$ & 0.00 & 1.01 \\
\hline Difficulties staying asleep & $0.4 I(0.99)$ & $1.21(1.33)$ & 0.00 & 0.68 \\
\hline ESS total & $5.11(4.35)$ & $6.23(5.48)$ & 0.27 & - \\
\hline PDQ total score & $27.11(22.28)$ & $43.79(27.53)$ & 0.00 & 0.67 \\
\hline
\end{tabular}

Notes: *Mean and standard deviation; ${ }^{\text {CCohen's } d .}$

Abbreviations: PD, Parkinson's disease; y, years; UPDRS, unified Parkinson's disease rating scale; LED, levodopa equivalent dose; MMSE, mini-mental state examination; PSQI, Pittsburgh sleep quality index; ESS, Epworth sleepiness scale; PDSS-2, PD sleep scale - second version; PDQ, PD Quality of life questionnaire.

affected by nocturnal disability, which is compatible with the findings from a previous study. ${ }^{29}$ It seems that nocturnal disturbances are a cross-cultural issue. ${ }^{21,30}$ Similar to studies from other countries, ${ }^{29}$ we found that the most common and disturbing nocturnal disturbance is "get up at night to pass urine", while more than half of the patients have "distressing dreams at night" and "difficulties falling asleep". When comparing two other studies, ${ }^{21,30}$ no significant differences were found in terms of PD symptoms at night between the

Table 3 Logistic regression analysis for determining the impact of different factors on PSQI $>5$

\begin{tabular}{llll}
\hline & OR & $\mathbf{9 5 \%} \mathbf{C I}$ & P-value \\
\hline No I subjective sleep quality & 2.044 & $\mathrm{I} .34 \mathrm{I}-3 . \mathrm{II} 4$ & $0.00 \mathrm{I}$ \\
No 2 difficulties falling asleep & 2.864 & $\mathrm{I} .54 \mathrm{I}-5.32$ & $0.00 \mathrm{I}$ \\
No 4 restlessness of legs/arms at night & $3.36 \mathrm{I}$ & $\mathrm{I} .32 \mathrm{I}-8.55$ & $0.0 \mathrm{II}$ \\
No 5 urge to move your legs or arms & 0.464 & $0.225-0.96$ & 0.038 \\
\hline
\end{tabular}

Abbreviations: PSQI, Pittsburgh sleep quality index; OR, odds ratio; $\mathrm{Cl}$, confidence interval; No, item number of the Parkinson's disease sleep scale - second version.
Table 4 Correlation coefficients between PDQ summary index and the variables analyzed

\begin{tabular}{lll}
\hline Variables & Correlation coefficients & P-value \\
\hline Age, y & 0.019 & 0.796 \\
Age of onset, y & -0.172 & 0.017 \\
Education, y & -0.050 & 0.497 \\
Disease duration, y & 0.431 & 0.000 \\
Hoehn and Yahr stages & 0.434 & 0.000 \\
MMSE & -0.168 & 0.020 \\
LED & 0.372 & 0.000 \\
UPDRS part I (mentality) & 0.501 & 0.000 \\
UPDRS part II (daily activities) & 0.559 & 0.000 \\
UPDRS part III (motor) & 0.273 & 0.000 \\
PDSS-2 total score & 0.526 & 0.000 \\
PSQI global score & 0.398 & 0.000 \\
ESS & 0.161 & 0.027 \\
\hline A & & Park
\end{tabular}

Abbreviations: PD, Parkinson's disease; y, years; UPDRS, unified Parkinson's disease rating scale; LED, levodopa equivalent dose; MMSE, mini-mental state examination; PSQI, Pittsburgh sleep quality index; ESS, Epworth sleepiness scale; PDSS-2, PD sleep scale - second version; PDQ, PD Quality of life questionnaire. 
Table 5 Linear regression analysis for determining the impact of clinical features and sleep problems on QoL

\begin{tabular}{|c|c|c|c|c|c|}
\hline & Beta & $\Delta R^{2}$ & Adjusted $R^{2}$ & $P$-value & $95 \% \mathrm{Cl}$ \\
\hline UPDRS2 & 0.231 & & 0.302 & 0.001 & $0.436-1.696$ \\
\hline Sleep disturbances** & 0.114 & 0.097 & 0.396 & 0.062 & -0.294 to 11.609 \\
\hline UPDRSI & 0.218 & 0.044 & 0.437 & 0.001 & $1.462-5.134$ \\
\hline Pain in arms or legs* & 0.223 & 0.039 & 0.474 & 0.000 & $3.376-9.566$ \\
\hline Daytime dysfunction** & 0.185 & 0.024 & 0.496 & 0.002 & $2.310-9.649$ \\
\hline Uncomfortable and immobility at night* & 0.177 & 0.025 & 0.519 & 0.003 & $0.992-4.720$ \\
\hline LED & 0.135 & 0.014 & 0.530 & 0.024 & $0.001-0.016$ \\
\hline
\end{tabular}

Notes: *PDSS-2; **PSQI; $\Delta R^{2}$, change in explained variance after adding the variable.

Abbreviations: UPDRS, unified Parkinson's disease rating scale; LED, levodopa equivalent dose; PSQI, Pittsburgh sleep quality index; PDSS-2, Parkinson's disease sleep scale - second version; QoL, quality of life; Cl, confidence interval.

three countries; however, Japanese patients had higher motor symptom scores than did our patients, and European patients had the highest disturbed sleep scores. One Japanese study indicated that there was a significant impact of comorbid rapid eye movement sleep behavior disorders on night-time disturbances and QoL in PD. ${ }^{31}$ Although nocturnal disabilities are common in PD patients regardless of country and race, this heterogeneity should be noted.

We found that poor sleepers spent more time falling asleep, had less actual sleep time, and had worse sleep efficiency. Poor sleepers experienced more nocturnal disturbances, including disturbed sleep (difficulties falling asleep and staying asleep) and motor symptoms at night (restlessness of the legs or arms at night), and they also had worse overall QoL. The predictors of poor night-time sleep quality for PD patients were included difficulties falling asleep and restlessness of the legs/arms at night. Louter et al, ${ }^{27}$ proposed that nocturnal hypokinesia is the main factor causing sleep disturbances; our results further suggest that the onset of insomnia and restlessness of legs at night are the most crucial sleep disturbances, and they ultimately merit therapeutic attention. Previous studies have shown that the prevalence of restless legs syndrome may be lower in Asian PD populations; ${ }^{32}$ nevertheless, our results suggest that restless legs syndrome plays an important role in sleep quality. In the logistic regression analysis, urge to move legs/arms was found to be a protective factor with odds ratio $(\mathrm{OR})<1(P=0.038)$. However, when comparing the scores of "urge to move legs/arms" between good and poor sleepers, no statistical significance was found. Considering the $P$-value being around 0.05 , and no statistical significance between good and poor sleepers, it suggests that "urge to move legs/arms" does not play a crucial in predicting the quality of sleep. We found a weak relationship between daytime somnolence and sleep quality at night, and EDS did not contribute to sleep quality at night in the predictive model. It seems that daytime and night-time sleep problems have distinct underlying mechanisms and require different forms of management.

Strong and significant correlations between QoL and nocturnal disturbances, daily motor experiences, and cognition or behavior were observed. After considering all of the possible predictors of QoL, several factors were identified as significant contributing factors, namely, daily motor experiences and mentality/mood dysfunction, sleep disturbances and daytime dysfunction, pain in the arms or legs and immobility at night, and LED. Our results are compatible with those from previous studies, and they emphasize the impact of sleep ${ }^{7,11,15}$ and other non-motor features ${ }^{33}$ on QoL. Interestingly, our results indicate that sleep disturbances at night hold critical importance for QoL, whereas daytime sleepiness does not seem to influence this outcome. Nocturnal disturbances such as sleep disturbances, pain in the arms or legs, and immobility at night have a negative impact on PD patients' QoL. As these are treatable conditions, they should be recognized by clinicians and managed properly. However, the direction of causality between sleep disturbances at night and daily QoL is often difficult to establish. The quantity of these relationships and how they correlate to the clinical heterogeneity in PD is not fully understood; thus, there is a need for longitudinal studies to examine the nature of the intertwined relationship between sleep problems and QoL in PD populations.

Some limitations exist in this study. First, only patients who were able to come to, and who were suitable for, the examination and interview were included. A number of patients with dementia were not included in our study, meaning that the sample is not representative of all PD patients. Despite this fact, this study still shows a high prevalence of sleep and nocturnal problems, resulting in worse QoL, so we expect that these factors will be even more disturbed in the total PD patient group, thus leading to an even worse QoL. Second, no control populations were recruited, and since the 
PSQI and PDSS-2 are disease-specific scales, there are no Taiwanese normative data available. Third, we found that "daytime dysfunction" is one predictor of QoL; however, apathy and depression might also influence QoL in our patients. The mentality/mood status of the patients has been globally addressed with the UPDRS and PSQI. We suggest that further studies use specific measures of neuropsychiatric symptoms, such as apathy and depression, to investigate how these symptoms can influence patients' QoL. Fourth, admittedly, due to the complicated medical conditions of these aged PD patients, confounding factors, such as physical conditions and medications, which could potentially interfere with these patients' sleep patterns, were not completely excluded. Last, the cross-sectional study design and the lack of an objective measurement of sleep disturbances (eg, polysomnography) are other important limitations that require further longitudinal and comprehensive measurement studies to explore the predictors of long-term outcomes of PD patients with respect to sleep disturbances.

\section{Conclusion}

The current findings call for an increased awareness of sleep problems in Oriental PD patients, and they confirm a close relationship between nocturnal disturbances and impaired QoL. Most of our patients have longer sleep latency and duration, and lower sleep efficiency. Sleep hygiene education programs or cognitive behavioral therapy are thus needed to correct these unhealthy sleep habits. They can also be used to address bedtime sleep restrictions, modify distorted thinking surrounding the negative impacts of insomnia, or reduce the level of anxiety associated with difficulties falling asleep. We believe that nocturnal disturbances have a great impact on QoL and the efforts to target nocturnal disturbances may have substantial therapeutic potential for improving QoL in PD patients.

\section{Acknowledgments}

We thank all of the patients who participated in this study, and we are grateful for the grant support from the National Science Council, Taipei, Taiwan (MOST 103-2410-H-006120-MY2 and MOST 103-2633-H-006-001).

\section{Disclosure}

The authors declare no conflicts of interest in this work.

\section{References}

1. Martinez-Martin P, Schapira AH, Stocchi F, et al. Prevalence of nonmotor symptoms in Parkinson's disease in an international setting; study using nonmotor symptoms questionnaire in 545 patients. Mov Disord. 2007;22(11):1623-1629.
2. Tandberg E, Larsen JP, Karlsen K. A community-based study of sleep disorders in patients with Parkinson's disease. Mov Disord. 1998; 13(6):895-899.

3. Yu RL, Wu RM, Tai CH, Lin CH, Cheng TW, Hua MS. Neuropsychological profile in patients with early stage of Parkinson's disease in Taiwan. Parkinsonism Relat Disord. 2012;18(10):1067-1072.

4. Yu RL, Wu RM. Social brain dysfunctions in patients with Parkinson's disease: a review of theory of mind studies. Transl Neurodegener. 2013; 2(1):7.

5. Yu RL, Wu RM, Chiu MJ, Tai CH, Lin CH, Hua MS. Advanced theory of mind in patients at early stage of Parkinson's disease. Parkinsonism Relat Disord. 2012;18(1):21-24.

6. Adler CH, Thorpy MJ. Sleep issues in Parkinson's disease. Neurology. 2005;64(12 suppl 3):S12-S20.

7. Havlikova E, van Dijk JP, Nagyova I, et al. The impact of sleep and mood disorders on quality of life in Parkinson's disease patients. J Neurol. 2011;258(12):2222-2229.

8. Suzuki K, Miyamoto T, Miyamoto M, et al. Excessive daytime sleepiness and sleep episodes in Japanese patients with Parkinson's disease. J Neurol Sci. 2008;271(1-2):47-52.

9. Tan EK, Lum SY, Fook-Chong SM, et al. Evaluation of somnolence in Parkinson's disease: comparison with age- and sex-matched controls. Neurology. 2002;58(3):465-468.

10. Gunn DG, Naismith SL, Lewis SJ. Sleep disturbances in Parkinson disease and their potential role in heterogeneity. $J$ Geriatr Psychiatry Neurol. 2010;23(2):131-137.

11. Borek LL, Kohn R, Friedman JH. Mood and sleep in Parkinson's disease. J Clin Psychiatry. 2006;67(6):958-963.

12. Svensson E, Beiske AG, Loge JH, Beiske KK, Sivertsen B. Sleep problems in Parkinson's disease: a community-based study in Norway. BMC Neurol. 2012;12:71.

13. Chung S, Bohnen NI, Albin RL, Frey KA, Muller ML, Chervin RD. Insomnia and sleepiness in Parkinson disease: associations with symptoms and comorbidities. J Clin Sleep Med. 2013;9(11): 1131-1137.

14. Neikrug AB, Maglione JE, Liu L, et al. Effects of sleep disorders on the non-motor symptoms of Parkinson disease. J Clin Sleep Med. 2013; 9(11):1119-1129.

15. Martinez-Martin P, Rodriguez-Blazquez C, Kurtis MM, Chaudhuri KR. The impact of non-motor symptoms on health-related quality of life of patients with Parkinson's disease. Mov Disord. 2011;26(3): 399-406.

16. Hughes AJ, Daniel SE, Kilford L, Lees AJ. Accuracy of clinical diagnosis of idiopathic Parkinson's disease: a clinico-pathological study of 100 cases. J Neurol Neurosurg Psychiatry. 1992;55(3):181-184.

17. Martinez-Martin P, Gil-Nagel A, Gracia LM, Gómez JB, MartinezSarriés J, Bermejo F. Unified Parkinson's disease rating scale characteristics and structure. Mov Disord. 1994;9(1):76-83.

18. Tzeng JI, Fu Y-W, Lin C-C. Validity and reliability of the Taiwanese version of the Pittsburgh Sleep Quality Index in cancer patients. Int $J$ Nurs Stud. 2012;49(1):102-108.

19. Ma H-I, Hwang W-J, Chen-Sea M-J. Reliability and validity testing of a Chinese-translated version of the 39-item Parkinson's disease questionnaire (PDQ-39). Qual Life Res. 2005;14(2):565-569.

20. Chen NH, Johns MW, Li HY, et al. Validation of a Chinese version of the Epworth Sleepiness Scale. Qual Life Res. 2002;11(8):817-821.

21. Trenkwalder C, Kohnen R, Högl B, et al. Parkinson's disease sleep scale - validation of the revised version PDSS-2. Mov Disord. 2011; 26(4):644-652.

22. Buysse DJ, Reynolds CF 3rd, Monk TH, Berman SR, Kupfer DJ. The Pittsburgh Sleep Quality Index: a new instrument for psychiatric practice and research. Psychiatry Res. 1989;28(2):193-213.

23. Brodsky MA, Godbold J, Roth T, Olanow CW. Sleepiness in Parkinson's disease: a controlled study. Mov Disord. 2003;18(6):668-672.

24. Ghorayeb I, Loundou A, Auquier P, Dauvilliers Y, Bioulac B, Tison F. A nationwide survey of excessive daytime sleepiness in Parkinson's disease in France. Mov Disord. 2007;22(11):1567-1572. 
25. Ferreira JJ, Desboeuf K, Galitzky M, et al. Sleep disruption, daytime somnolence and 'sleep attacks' in Parkinson's disease: a clinical survey in PD patients and age-matched healthy volunteers. Eur J Neurol. 2006;13(3):209-214.

26. Johns MW. Reliability and factor analysis of the Epworth Sleepiness Scale. Sleep. 1992;15(4):376-381.

27. Louter M, Munneke M, Bloem BR, Overeem S. Nocturnal hypokinesia and sleep quality in Parkinson's disease. J Am Geriatr Soc. 2012; 60(6):1104-1108.

28. Yu RL, Wu RM, Tai CH, Lin CH, Hua MS. Feeling-of-knowing in episodic memory in patients with Parkinson's disease with various motor symptoms. Mov Disord. 2010;25(8):1034-1039.

29. Bhidayasiri R, Mekawichai P, Jitkritsadakul O, et al. Nocturnal journey of body and mind in Parkinson's disease: the manifestations, risk factors and their relationship to daytime symptoms. Evidence from the NIGHT-PD study. J Neural Transm. 2014;121(suppl 1):S59-S68.
30. Suzuki K, Miyamoto M, Miyamoto T, et al. Nocturnal disturbances and restlessness in Parkinson's disease: using the Japanese version of the Parkinson's disease sleep scale-2. J Neurol Sci. 2012; 318(1-2):76-81.

31. Suzuki K, Miyamoto T, Miyamoto M, et al. Probable rapid eye movement sleep behavior disorder, nocturnal disturbances and quality of life in patients with Parkinson's disease: a case-controlled study using the rapid eye movement sleep behavior disorder screening questionnaire. BMC Neurol. 2013;13(1):18.

32. Tan EK, Seah A, See SJ, Lim E, Wong MC, Koh KK. Restless legs syndrome in an Asian population: a study in Singapore. Mov Disord. 2001; 16(3):577-579.

33. Martínez-Martín P, Rodríguez-Blázquez C, Forjaz MJ, et al. Relationship between the MDS-UPDRS domains and the health-related quality of life of Parkinson's disease patients. Eur J Neurol. 2014;21(3): $519-524$

\section{Publish your work in this journal}

Neuropsychiatric Disease and Treatment is an international, peerreviewed journal of clinical therapeutics and pharmacology focusing on concise rapid reporting of clinical or pre-clinical studies on a range of neuropsychiatric and neurological disorders. This journal is indexed on PubMed Central, the 'PsycINFO' database and CAS, and is the official journal of The International Neuropsychiatric Association (INA). The manuscript management system is completely online and includes a very quick and fair peer-review system, which is all easy to use. Visit http://www.dovepress.com/testimonials.php to read real quotes from published authors. 